\title{
El cine como herramienta para la formación de traductores jurídicos
}

\author{
EIVOR JORDÀ MATHIASEN \\ Universidad Europea de Valencia \\ mariaeivor.jorda@uem.es
}

Recibido: 30 de septiembre de 2014

Aceptado: 15 de abril de 2015

\section{RESUMEN}

El movimiento "cine y derecho" se ha afianzado como una metodología innovadora en los estudios de derecho. Los resultados de esta disciplina pueden trasladarse a la enseñanza del derecho a estudiantes de traducción en la medida en que el visionado y posterior comentario de películas sobre temática jurídica puede resultar un modo atractivo de adquirir conocimientos de derecho. Con este objetivo, el presente artículo pretende servirse de algunas aportaciones de las investigaciones más recientes sobre cine y derecho para incluirlas en la formación de traductores jurídicos centrándonos en determinadas cuestiones jurídicas (como el propio concepto de derecho, los fundamentos generales del derecho y el derecho comparado). La investigación se completa con la presentación de cuatro ejemplos de análisis fílmico desde la perspectiva de la formación de traductores jurídicos, así como con un listado de películas adecuadas a este fin y clasificadas por la temática que abordan.

Palabras clave: Cine y derecho, traducción jurídica, formación de traductores, derecho comparado, cine y traducción.

\section{Film as a Tool in Legal Translation Training}

\begin{abstract}
The "Law and Cinema" movement has established itself as an innovative methodology in Law Studies. The results of this discipline can be transferred to the teaching of law to translation students, since the viewing and discussion of films with legal topics can appeal and motivate translation students in their training. To contribute to such purpose, this paper presents topics of recent film and law research, and proposes ways to include them in legal translation training, focusing on relevant legal issues (such as the concept of law itself, principles of law, civil versus criminal law, procedural law and comparative law). The research is completed with a list of legal films -classified by legal topic- that can be used as an introduction of those questions in the classroom, and an explanation of four


examples of film analysis from the perspective of legal translation training, with special emphasis on comparative law.

Keywords: Law and cinema, legal translation, legal translation training, comparative law, cinema and translation.

Sumario: 1. Introducción. 2. Cine y derecho. 3. El cine en los estudios de derecho. 4. La formación jurídica de los traductores. 5. El cine en la formación de traductores jurídicos. 6 . Cuatro ejemplos. 6.1. Doce hombres sin piedad. 6.2. Anatomía de un asesinato. 6.3. Vencedores o vencidos. 6.4. Veredicto final. 7. Conclusiones.

\section{Introducción}

La traducción jurídica es una disciplina que requiere de la adquisición de ciertos conocimientos de derecho. La particularidad de esta especialidad de la traducción estriba en que los conceptos difieren a menudo entre los distintos sistemas jurídicos, por lo que se producen frecuentes situaciones de inequivalencia. No obstante, los estudiantes de traducción jurídica no necesitan profundizar en la materia del mismo modo que los estudiantes de derecho. Por este motivo, nos encontramos ante la exigencia de encontrar metodologías innovadoras para el aprendizaje de ciertas nociones jurídicas por parte de los estudiantes de traducción.

El análisis de la relación entre el cine y el derecho se ha introducido recientemente en el contexto de los estudios de derecho. En las últimas décadas, los resultados de estas investigaciones han tenido una aplicación directa en las universidades de muchos países (como Estados Unidos o España). Así y todo, cabe señalar que esta metodología innovadora para transmitir conocimientos de derecho no está exenta de algunos inconvenientes, como la dificultad para distinguir entre ficción y realidad en los textos cinematográficos.

El principal objetivo del presente artículo es tratar de aplicar los resultados de algunos estudios clave sobre cine y derecho (como los de Machura y Robson 2001, Chase 2002, Levi 2005, Presno y Rivaya 2006 o Greenfield, Osborn y Robson 2010) a la formación de estudiantes de traducción jurídica. Con este propósito, presentaremos algunas ideas desarrolladas por el denominado movimiento "cine y derecho", que puedan ser de utilidad para su aplicación a nuestra área. En resumidas cuentas, las principales cuestiones que los estudiantes de traducción deberían plantearse con respecto al derecho son: ¿Qué es el derecho? ¿Quiénes son los principales agentes en el procedimiento judicial? ¿El derecho es el mismo en todos los países?

\section{Cine y derecho}

En uno de los principales trabajos sobre cine y derecho, Machura y Robson (2001: 3-8) afirman que, a finales de los años 80 y principios de los 90, algunas publicaciones académicas pioneras se embarcaron en un proyecto que combinaba 
los estudios de derecho con los del cine, la narrativa cinematográfica y la imaginería visual popular. Desde entonces, la idea de que se pueden establecer puentes entre el estudio del derecho y el del cine ha ido obteniendo cada vez más relevancia en ciertas universidades europeas y estadounidenses.

En otra destacable obra de Greenfield, Osborn y Robson (2010: 3-8), los autores describen las diferentes vías de aproximación a esta materia. En una fase inicial, los estudios sobre cine y derecho se centraron en la confrontación entre el derecho "real" y el derecho que aparece en las películas, con el fin de poner de relieve las "imprecisiones". Más tarde, se prestó mayor atención a las consecuencias de la disparidad entre lo que se proyecta en las pantallas y la práctica jurídica real, es decir, a la importancia de los estereotipos que se transmiten a través del cine. En una fase más reciente, la corriente de cine y derecho se ha ocupado en describir el modo en que las películas pueden forjar nuestras expectativas sobre el derecho y la justicia (Silbey 2009: 756-757). Como último enfoque de esta línea, Greenfield, Osborn y Robson (2010: 7) señalan el análisis del paralelismo entre la construcción narrativa del discurso jurídico y la forma narrativa de las películas.

\section{El cine en los estudios de derecho}

Las películas pueden ser un buen punto de arranque para reflexionar sobre el derecho. Tal y como apuntan Presno y Rivaya (2006: 19-27), se trata de un modo innovador de transmitir conocimientos de derecho colocando al estudiante en el centro del proceso de aprendizaje. No obstante, la utilización de películas para analizar cuestiones jurídicas no está exenta de ciertas dificultades. La principal de ellas es la simplificación de los temas jurídicos que se produce en los textos fílmicos como consecuencia del tratamiento dramatizado de la práctica jurídica. Por este motivo, resulta aconsejable ofrecer materiales complementarios y promover el debate en el aula.

No obstante, cabe incidir en que, por lo que respecta a la relación entre realidad y ficción en el cine, resulta irrelevante el hecho de que la construcción cultural mostrada en la pantalla sea falsa, ya que lo verdaderamente relevante es el ideal de justicia mostrado (véase Chase 2002). Tal y como concluyen Greenfield, Osborn y Robson (2010: 176): "There is a balance to be struck with such films as they are often an uneasy compromise between the "truth" and the cinematic equivalent".

A pesar de ello, el cine puede ser de gran utilidad para el aprendizaje de conceptos jurídicos ya que nos permite ilustrar cuestiones concretas como el desarrollo de los procesos judiciales o la función que cumplen los distintos agentes judiciales (jueces, abogados, jurado o fiscales). Además, el cine nos ayuda a centrar la atención en cuestiones éticas relevantes como la pena capital, la discriminación laboral, las negligencias médicas, el racismo, los abusos sexuales y la homofobia, entre otros. A este respecto, las películas pueden servir de desencadenante de un debate en el aula con el fin de promover el interés de los estudiantes hacia estos temas. En el anexo ofrecemos un listado de películas clasificadas según la temática jurídica que abordan. 


\section{La formación jurídica de los traductores}

La traducción jurídica suele englobarse dentro de la rama de la traducción especializada en tanto que el traductor debe poseer ciertos conocimientos de la materia. Sin embargo, existe una clara diferencia entre la traducción jurídica y otras subdisciplinas de la traducción especializada, como la traducción científica. En este último caso, los referentes de los términos son conceptos de carácter universal; por el contrario, suele darse el caso de que los conceptos contenidos en un texto jurídico pueden no contar con un equivalente en la cultura meta.

Tal y como señala Monzó (2008), el traductor jurídico debe familiarizarse con las cuestiones jurídicas más generales, conocer las ramas específicas del derecho y la función que desempeñan los distintos agentes que operan en la administración de la justicia. En este sentido, cualquier módulo teórico en un curso de formación de traductores jurídicos debería abordar cuestiones como el concepto del derecho, los principios generales del derecho, el derecho procesal, el derecho civil, el derecho penal, el derecho mercantil, el derecho internacional y el derecho comparado. El derecho comparado, en concreto, es de especial relevancia en la medida en que, en traducción jurídica, resulta más apropiado hablar de la traducción de sistemas jurídicos que de la traducción de palabras (Šarcevic 1994: 303).

Si comparamos, por ejemplo, la familia jurídica anglo-americana (conocida como "derecho común" o "derecho casuístico") y la familia continental europea (conocida como "derecho continental" o "derecho codificado"), podemos observar que la distinta concepción del derecho que se deriva de estos dos grandes sistemas determina el uso del lenguaje en los respectivos textos jurídicos. En el primer caso, los fallos de los jueces tienen un carácter normativo y están basados en precedentes; por ello, no resulta indispensable que los sistemas legislativos sean exhaustivos (Altava 2003: 49). En el segundo caso, la legislación es la principal fuente del derecho y, como consecuencia de ello, los sistemas jurídicos codificados exigen una estructuración sistemática y completa de las normas generales del derecho en grandes sistemas conceptuales (véase Zweiegert 1996).

Estas diferencias determinan el carácter más argumentativo y conciso del lenguaje jurídico propio de los sistemas anglo-americanos, frente a un carácter más conceptual y abstracto del lenguaje jurídico en los sistemas continentales europeos. Por esta razón, se puede afirmar que: "It would be a mistake to underestimate the linguistic difficulties this situation causes translators" (Alcaraz y Hughes 2002: 47).

\section{El cine en la formación de traductores jurídicos}

En el apartado anterior, hemos tratado de argumentar que para la formación de traductores jurídicos resulta indispensable la adquisición de ciertos conocimientos de derecho. Para este propósito, la aplicación del movimiento "cine y derecho" a la formación de juristas ha allanado el camino para la utilización de películas con fines académicos. En palabras de Kamir (2005: 275): "Films have a unique way of touching people's hearts and allowing them to employ their emotions in the process of seeing, listening, and understanding, discussing and analyzing". 
No obstante, debemos ser cautelosos por lo que respecta a la compleja relación entre realidad y ficción en el discurso cinematográfico, en los términos comentados en el apartado 2 de este artículo. Por ello, las películas deben interpretarse como una expresión del ideal de justicia -tanto si se postulan a favor como en contra del sistema establecido - con capacidad para distorsionar la percepción del sistema jurídico retratado en la pantalla pero también para transformar la concepción futura del derecho de los espectadores.

Curiosamente, la preocupación por la relación entre realidad y ficción cada vez está más presente en los estudios de traducción. En esta línea, la traducción está dejando de ser concebida como una actividad pura, neutral o invisible, y ha pasado a entenderse como una interpretación, recreación o incluso manipulación del texto origen. Diversos autores y autoras, como Bassnet (2013), Venuti (2008), Baker (2006), Lefevere (2002) Gentlzer (2001), Tymoczko (1999), Hermans (1999), Godard (1990) o Derrida (1985) comparten este punto de vista.

En este sentido, tal y como apunta Vidal (2005), los conceptos de "re-presentación" y realidad son de extrema importancia en la traducción jurídica. Hemos de tener en cuenta que, desde un enfoque postestructuralista, la realidad no puede concebirse como algo externo a las "re-presentaciones". Desde esta perspectiva resulta evidente que el modo en que un texto es interpretado necesariamente genera nuevos significados que, a su vez, reestructurarán la percepción de la realidad. "Approaches to legal translation must thus conceive of the real as dependent on the interpretation and the re-presentation made of it through language" (Vidal 2005: 262).

Además, las películas pueden servirnos también como un modo de ejemplificar estas relaciones de poder. En primer lugar, el texto cinematográfico nos puede ilustrar sobre el sentido de determinados conceptos del derecho, como la contraposición entre justicia y ley, los derechos humanos, el derecho civil frente al derecho penal, el derecho procesal, etcétera. En segundo lugar, el cine también puede ser útil como punto de partida para generar un debate sobre cuestiones más específicas como: la función que cumple el jurado, el sentido (o sinsentido) de la pena capital o la cadena perpetua, o la indemnización como compensación de unos daños. En tercer lugar, las películas pueden ayudar a examinar las diferencias entre los distintos sistemas jurídicos y remarcar su carácter cultural.

\section{Cuatro ejemplos}

En este apartado vamos a analizar cuatro películas como ejemplo de cómo se pueden aplicar las ideas del movimiento "cine y derecho" a la formación jurídica de traductores. En la medida en que los traductores no necesitan acercarse al derecho del mismo modo que los juristas, el presente estudio se centra en los aspectos comentados en el apartado 3. Abordaremos primero cuestiones generales del derecho que se pueden ver reflejadas en las cintas y que pueden ejercer de objeto de un debate posterior en el aula. Además, prestaremos atención a la manera en que abogados, jueces y jurados son retratados, pero teniendo en cuenta que en la mayoría de ocasiones nos encontramos ante un retrato estereotipado. Por último, presentaremos 
algunos elementos de la película que pueden servir para ilustrar ciertas cuestiones sobre derecho comparado.

\subsection{Doce hombres sin piedad}

Esta película es considerada por muchos la mejor cinta sobre cuestiones jurídicas, ya que capta de manera brillante la dinámica interna del derecho, es decir, la argumentación jurídica y la justificación de las decisiones. En este sentido, el cometido del jurado resulta ser el de la argumentación mediante la palabra. Como Rivaya (2012: 79) señala, en este filme, el discurso jurídico adquiere un sentido dialógico, según el cual el objetivo es convencer a los demás de las propias decisiones (frente a la concepción monológica del derecho, donde la práctica del mismo se apoya en un razonamiento subjetivo basado en las normas del derecho).

Doce hombres sin piedad es un alegato en favor del buen funcionamiento de la justicia, una visión optimista del derecho y una defensa apasionada del jurado como institución. Pudiera parecer que en algún momento se pone en duda la supuesta infalibilidad del sistema al destacar algunas debilidades del jurado como la fuerza de la costumbre, la ignorancia o la tendencia gregaria del ser humano, que podrían haber llevado al jurado a tomar una decisión sin una reflexión previa. Sin embargo, cuando el personaje principal expone sus dudas sobre la culpabilidad del acusado y expresa la necesidad de "hablar", mediante este gesto activa el mecanismo de la argumentación (véase Soto y Fernández 2004).

Además, en esta película se analiza minuciosamente el funcionamiento del jurado, haciendo especial hincapié en la responsabilidad del individuo frente a la sociedad. Puesto que la justicia no se puede resolver mediante una mera decisión técnica, ni se puede alcanzar si no nos deshacemos de los prejuicios sociales, la ética es presentada en el filme como un compromiso personal. Para que el sistema funcione correctamente, el individuo debe adoptar una actitud positiva y activa; una actitud contraria a la pasividad generalizada que espera del sistema que resuelva los problemas por sí mismo (véase Soto y Fernández 2004).

Otro tema jurídico que se puede explorar en esta película es el concepto de prueba en un procedimiento judicial. La conclusión a la que llegan los propios miembros del jurado en Doce hombres sin piedad es que solo puede quedar probado aquello sobre lo que no existe ninguna "duda razonable". Tras una larga discusión resulta que ninguna prueba puede sostenerse, pero esa es precisamente la misión de la justicia: construir un discurso fiable, coherente, completo y comprensible sobre los hechos juzgados. Debe explicarse con claridad tanto la decisión tomada como la inaceptabilidad de cualquier otra explicación posible (Rivaya 2012: 82). En otras palabras, la verdad en un procedimiento penal es básicamente argumentativa en tanto que es una cuestión meramente probabilística en términos de razonabilidad (las pruebas son únicamente hechos presentes que nos permiten interpretar hechos pasados). Por ello, el discurso jurídico se sitúa en el marco de las garantías para posibilitar la igualdad de oportunidades (Latorre 2005: 34). 
Por lo que respecta al retrato de los agentes judiciales, esta película es clave para entender la función del jurado en el sistema judicial estadounidense. Doce hombres sin piedad es, de hecho, un compendio de personajes típicos en la composición de los jurados. En cambio, el juez, que solo aparece en la primera escena de la cinta y es retratado como un juez ausente "who has had contact with too many courtroom antics, loathsome clients and misapplications of justice" (Levi 2005: 51), es presentado como un personaje periférico en la medida en que el jurado es quien simboliza la justicia en esta cinta.

En el caso del abogado defensor, nos encontramos ante un abogado de oficio que no se esfuerza demasiado en su cometido. Con esto se pone de relieve que una mala defensa supone una grave desventaja para el acusado ya que no se activan todos los mecanismos para desarrollar la mejor estrategia que garantice un juicio justo. Tal y como afirma el protagonista: "He really has to believe in his client to put up a good case. Obviously he didn't". Este es nuevamente otro ejemplo de la importancia de la argumentación en los procedimientos jurídicos; también lo es de la importancia de que para respetar el principio "in dubio pro reo" (la presunción de inocencia) la carga de la prueba debe correr a cargo de la acusación.

Doce hombres sin piedad da pie a comentar dos cuestiones concretas sobre derecho comparado. En primer lugar, se subraya la posición no central de los jueces en el sistema judicial estadounidense, sobre todo en comparación con los sistemas continentales europeos. La tradición del derecho común inglés determina que el juez se ocupe fundamentalmente de las cuestiones de derecho pero deje en manos del jurado las cuestiones de hecho. Además, en los sistemas continentales europeos el juez es el encargado de dirigir la instrucción penal (tarea asignada a la fiscalía en los sistemas anglo-americanos) (Greenfield, Osborn y Robson 2010: 125-133).

En segundo lugar, se plantea la cuestión de la conveniencia del jurado, al que el sistema judicial estadounidense concede mayor peso que los sistemas de los países europeos. La postura a favor del jurado se fundamenta en la idea de que el poder judicial debe democratizarse al igual que el resto de los poderes del estado (legislativo y ejecutivo). Por este motivo, el jurado es defendido como una institución que permite a los ciudadanos participar en las decisiones judiciales que no requieren de conocimientos técnicos. Los defensores del jurado sostienen además que se reducen las posibilidades de corrupción y que los jueces se acaban insensibilizando ante los problemas personales o sociales de las partes. Las posturas contra el jurado afirman que en los procedimientos judiciales se deben tener en cuenta cuestiones técnicas que solo los juristas conocen así como que los jurados son más fácilmente influenciables (por abogados, testigos o, incluso, los medios de comunicación) (véase Soto y Fernández 2004).

\subsection{Anatomía de un asesinato}

En Anatomía de un asesinato podemos examinar igualmente la concepción de la actividad jurídica como una aplicación práctica de las normas positivas. Los jueces y los jurados se nos muestran como seres humanos en toda su complejidad y no 
como meros entes racionales. En consecuencia, los personajes actúan conforme a todo aquello que mueve al ser humano, no solo los argumentos, sino también los prejuicios o las emociones (Rivaya 2012: 95). Esto se ilustra en un momento dado en el filme cuando el acusado pregunta a su abogado: "How can a jury disregard what it has already heard?" a lo que el abogado responde: "They can't, Lieutenant, they can't'.

En la batalla argumentativa, el objetivo de los abogados defensores es encontrar una "percha legal", es decir, un argumento jurídico para convencer al jurado de que existe alguna circunstancia eximente a una conducta que el jurado está en disposición de perdonar. Su principal problema es que el argumento comúnmente utilizado (la incapacidad del acusado para distinguir entre el bien y el mal) no se puede alegar en ese caso. Por este motivo, los abogados defensores se concentran en la búsqueda de un precedente (que finalmente encuentran en una sentencia del Tribunal Supremo de Michigan de 1886).

Esta película resulta muy útil para comentar algunas cuestiones sobre derecho comparado, ya que refleja bastantes particularidades del sistema judicial de Estados Unidos. En primer lugar, tenemos el uso del precedente como principal fuente del derecho (como acabamos de mencionar). Otra diferencia con respecto a los sistemas jurídicos codificados es la inexistencia de la acusación particular en los procedimientos penales; en estos casos, la fiscalía se considera la única defensa posible de la legalidad. La tercera disimilitud que se puede observar es la diferente forma de acceso a los puestos de la administración de justicia. En Anatomía de un asesinato vemos cómo el fiscal es elegido por un cierto periodo de tiempo, como también sucede en algunos estados en el caso de los jueces.

En contraposición a Doce hombres sin piedad, el jurado ocupa en esta película una posición muy secundaria, y realmente solo lo vemos actuar cuando emite el veredicto. Sin embargo, en esta cinta aparece uno de los pasajes más emotivos en defensa del jurado cuando uno de los abogados define el "misterio del jurado":

Twelve people go off into a room. Twelve different minds, twelve different hearts, twelve different walks of life. Twelve sets of eyes, ears, shapes and sizes. And these twelve people are asked to judge another human being as different from them as they are from each other. And in their judgement they must become of one mind... unanimous. That's one of the miracles of man's disorganized soul that they can do it, and in most instances do it right well. God bless the juries!

\subsection{Vencedores o vencidos}

Vencedores o vencidos es una película en la que queda reflejado el hito histórico que supuso el juicio de Nuremberg, en el que se examinaron cuestiones relativas a la complicidad individual en crímenes cometidos por los estados a los que sirven. Tal y como establecen Muñoz y Muñoz (2003), las acusaciones que se formularon contra los jueces alemanes fueron las siguientes: 
- Cargos por crímenes de guerra por la creación de tribunales especiales y leyes con el propósito de confinar a determinadas personas en campos de concentración y exterminarlas por pertenecer a ciertas minorías étnicas (judíos, polacos, gitanos) o ser individuos "asociales" (homosexuales, alcohólicos, disidentes políticos).

- Cargos por crímenes contra la humanidad por la creación de los "Tribunales de Salud Hereditaria" para aplicar la Ley de Esterilizaciones de 1933, no solo para prevenir enfermedades hereditarias (tal y como preveía la ley), sino para librarse de los denominados "asociales".

- Cargos por la aplicación de la Ley para la Protección de la Sangre y el Honor Alemanes de 1935 con el fin de penalizar las relaciones sexuales entre judíos y arios.

Esta película trata fundamentalmente de la aplicación de leyes injustas y de la responsabilidad de los jueces que las aplican. Tal y como el fiscal señala, son "crímenes cometidos en nombre de la ley"; por ello, su discurso se basa en el argumento de que los jueces alemanes pervirtieron el sentido de la justicia y de las leyes de forma injustificada. En este sentido, el tema de la película puede entenderse como el problema ético fundamental de los límites del estado en el uso de la ley. A este respecto Insdorf comenta (1983: 170): "The film raises central issues of responsibility-individual, national, universal".

Esta cuestión de la responsabilidad de los ciudadanos se puede vincular a las dos concepciones contrapuestas entre derecho positivo y derecho natural. Por un lado, los defensores del derecho positivo argumentan que no hay otra ley que las normas existentes en cada territorio en un momento dado (Rivaya 2012: 101-102). En Vencedores o vencidos esta es la perspectiva de la defensa, tal y como uno de los jueces acusados la formula en su alegato final:

I have served my country throughout my life, and in whatever position I was assigned to, in faithfulness, with a pure heart and without malice. I followed the concept that I believed to be the highest in my profession, the concept that says: to sacrifice one's own sense of justice to the authoritative legal order, to ask only what the law is, and not to ask whether or not it is also justice. As a judge, I could do no other. I believe Your Honours will find me, and millions of Germans like me, who believed they were doing their duty to their country, to be not guilty.

Por otro lado, la fiscalía defiende en este juicio la idea del derecho natural, es decir, que ley y ética son inseparables en la medida en que los derechos universales del hombre son irrenunciables (De Domingo 2008: 68).

\subsection{Veredicto final}

En Veredicto final encontramos otra versión de la idea de que el cumplimiento de la justicia corresponde a personas individuales (como comentábamos a pro- 
pósito de Doce hombres sin piedad). Aquí se plantea de nuevo la cuestión de la relación entre justicia (el instrumento utilizado por la sociedad para garantizar la igualdad) y ética (la percepción individual de lo que es justo). No obstante, Veredicto final se rodó veinticinco años después de Doce hombres sin piedad y la justicia ya no se muestra con tanto idealismo sino plagada de imperfecciones (la desigualdad económica entre las partes, los intereses creados, las estrategias de los abogados o, incluso, la corrupción). Así y todo, la conclusión sigue siendo optimista y, aunque se remarcan las "debilidades" humanas, insiste en la posibilidad de que se imparta justicia. Como el abogado de la acusación argumenta al dirigirse al jurado:

Today you are the law. You are the law... Not some book, not the lawyers, not a marble statue or the trappings of the court. See, those are just symbols of our desire to be just. [...] If we are to have faith in justice, we need only to believe in ourselves and act with justice.

Esta película nos sirve de ejemplo de un procedimiento civil: una reclamación de indemnización por daños debido a una negligencia médica. La cuestión sobre la conveniencia o no de compensar un daño mediante una cantidad de dinero se aborda en varios momentos del filme. En una conversación con el demandado, por ejemplo, el abogado de la acusación pregunta en qué se han basado para calcular la suma que ofrecen para intentar llegar a un acuerdo (Soto y Fernández 2004: 387).

Otra cuestión jurídica que se trata en Veredicto final es el concepto de imprudencia profesional. En nuestra sociedad hemos asumido que las personas son responsables de las consecuencias de todos sus actos (en la medida en que hemos abandonado el sentido fatalista de la vida). Por ello, los individuos que cometen errores deben reconocer su responsabilidad y compensar a la víctima. No obstante, esto puede resultar complejo en un caso médico donde hay múltiples factores en juego y no todos dependen del buen ejercicio profesional.

También puede comentarse a propósito de esta cinta la cuestión de la carga de la prueba. La carga de la prueba en los procedimientos civiles corresponde al demandante; por ello el abogado acusador de esta historia debe presentar las pruebas para poder atribuir la negligencia al equipo médico. Esto coloca al paciente en una situación complicada, al resultarle difícil acceder a los documentos. Otra complejidad añadida, incluso cuando existen pruebas de una negligencia médica, es la tecnicidad de los términos médicos. Por ello los tribunales suelen nombrar peritos para que expliquen la materia tanto al propio tribunal como al jurado (De Madaria 2008: 57).

Por lo que respecta al derecho comparado, esta película nos sirve para profundizar en las diferentes formas de entender el concepto de responsabilidad. En los sistemas de derecho codificado como el nuestro, se distingue entre responsabilidad civil y penal; es decir, la ley establece, por un lado, un deber de compensación a la víctima y, por otro, una pena por la comisión de un delito. En contraposición, en los sistemas de derecho común, como el estadounidense, la imprudencia no se contempla en el derecho penal (Fletcher 1998: 49). 


\section{Conclusiones}

El objetivo del presente artículo ha sido aplicar algunas consideraciones de las investigaciones sobre cine y derecho llevadas a cabo en diversas universidades de Estados Unidos y España desde finales de la década de 1980. Tal y como hemos señalado en los apartados 1 y 2, la utilización de películas para explorar conceptos jurídicos resulta muy productiva en la medida en que la confluencia de ambas disciplinas permite enfoques muy diversos. Entre estos enfoques podemos encontrar los estudios sobre la relación entre realidad y ficción en el cine, el análisis de los estereotipos transmitidos a través de las películas (estereotipos que pueden a su vez transformar nuestra percepción sobre derecho y justicia) y la comparación entre el discurso narrativo construido por los juristas y el del cine.

Es cierto que la principal dificultad de esta metodología para el aprendizaje de conceptos legales es la posible confusión entre realidad y ficción, que constituye la esencia del cine. No obstante, esta circunstancia debe contemplarse desde la perspectiva de que las películas suponen una idealización del mundo real, por lo que sirven tanto para crear un discurso sobre nuestra concepción actual del derecho como para transformarla. Esta conclusión es comparable con algunas corrientes actuales en traductología que se oponen a la idea de la traducción como un proceso meramente técnico y neutral, y defienden el carácter interpretativo y re-creativo de la traducción.

Hemos comentado también que en la formación de traductores jurídicos no solo se deben tener en cuenta cuestiones de estrategias de traducción generales, sino que resulta imprescindible una cierta base de conocimientos jurídicos. Además, puesto que la disciplina jurídica no trabaja con conceptos universales sino marcados culturalmente, el traductor jurídico debe familiarizarse con el derecho comparado. La aproximación al derecho por parte del traductor jurídico no exige la sistematicidad y profundidad de los conocimientos de un jurista, aunque deberá formarse una idea clara de lo que es el derecho, de cuáles son sus principios generales y de algunas nociones básicas referentes a las principales ramas del derecho.

Con el presente artículo se ha pretendido mostrar que el cine puede servir como herramienta para introducir a los estudiantes de traducción en el derecho de un modo sencillo y ameno. En el listado de películas sobre derecho incluido como anexo, se ofrece una muestra de los filmes más relevantes clasificados por ramas del derecho: filosofía del derecho, derecho constitucional, derecho procesal, derecho penal, derecho civil, derecho mercantil, derecho público internacional, derecho privado internacional, derecho del trabajo y derecho del medio ambiente. Mediante esta selección, el docente de traducción jurídica puede centrarse en temas concretos en el aula y utilizar las películas como introducción a una determinada materia.

En la última sección de este artículo hemos visto cuatro ejemplos de análisis de las películas Doce hombres sin piedad, Anatomía de un asesinato, Vencedores o vencidos y Veredicto final. La primera de ellas, Doce hombres sin piedad nos sirve como excusa para reflexionar sobre la naturaleza argumentativa del derecho. Tal y como se ha comentado, este filme refleja a la perfección la centralidad del lenguaje en el mundo jurídico, donde el razonamiento y la justificación de las decisiones con- 
figuran la esencia del derecho. Esta película también nos ilustra sobre el papel de la institución del jurado en el contexto de los Estado Unidos. Vemos al jurado como símbolo del espíritu democrático de dicha sociedad en el sentido de que permite a los ciudadanos participar en las decisiones judiciales. Otros temas de debate a los que puede dar pie Doce hombres sin piedad son la responsabilidad individual ante la sociedad y el concepto de prueba en relación al principio de presunción de inocencia.

Anatomía de un asesinato recalca las debilidades humanas (los prejuicios y las emociones) presentes en los procedimientos judiciales. En este sentido, la maestría en el uso del lenguaje (la elocuencia y la argumentación) resulta ser imprescindible para los abogados con el fin de controlar las debilidades del jurado. Puesto que esta película describe todas las fases de un procedimiento judicial, resulta un instrumento adecuado para comparar el derecho procesal estadounidense y el español. En ella vemos ejemplos del uso del precedente como fuente del derecho, la relevancia del jurado, la función del juez como árbitro o la elección de los cargos de la administración de justicia.

En Vencedores o vencidos, el tema principal es la responsabilidad de los jueces en la aplicación de leyes injustas o, dicho de otro modo, los límites del estado en el uso de la ley. Esta cuestión está directamente relacionada con la clásica controversia entre derecho positivo y derecho natural, es decir, entre la idea de que las normas creadas por una sociedad determinada es la única ley aplicable como contraposición a la idea de que existen unos derechos humanos que toda legislación está obligada a respetar.

Veredicto final se ha escogido como ejemplo de un procedimiento civil en el que además podemos analizar la complejidad del concepto de indemnización por daños (la dificultad de medir el daño, la carga de la prueba o la negligencia profesional). Por lo que respecta al derecho comparado, hemos comentado la diferente concepción de la responsabilidad entre los sistemas del derecho común y el derecho codificado (concebida exclusivamente como responsabilidad civil en el primer caso y tanto civil como penal en el segundo).

Como conclusión podríamos decir que, del mismo modo que el cine ha mostrado su utilidad como herramienta para el aprendizaje de los estudiantes de derecho, también puede servirnos como metodología innovadora para introducir conceptos de derecho en los estudios de traducción. Tal y como hemos tratado de subrayar en este artículo y citando a Champoux (1999: 207): "Cinema's ability to create a unique experience gives it unbeatable power as a teaching tool".

\section{Referencias bibliográficas}

Alcaraz Varó, E., y B. Hughes, Legal Translation Explained. Manchester: St. Jerome 2002. Altava Lavall, M. G., Lecciones de derecho comparado. Castelló de la Plana: Universitat Jaime I 2003.

BAKER, M., Translation and Conflict. A Narrative Account. Londres y Nueva York: Routledge 2006. 
BAssnett, S., Translation. Londres y Nueva York: Routledge 2013.

Champoux, J. E., «Film as a teaching resource», Journal of Management Inquiry 8-2 (1999), 206-217.

Chase, A., Movies on Trial: The Legal System on the Silver Screen. Nueva York: The New Press 2002.

Clover, C. J., «Movie juries», DePaul Law Review 48-2 (1998), 389-405.

De Domingo PÉrez, T., «Los jueces ante las leyes injustas. Algunas reflexiones sobre la película Vencedores o vencidos», en: A. Ortega Jiménez y P. Cremades García (eds.), Cine y derecho en 13 películas. Alicante: Editorial Club Universitario, 2008, 63-74.

DE MADARIA RUVIRA, J., «Una aproximación a la responsabilidad civil médica y sanitaria a propósito de la película Veredicto final», en: A. Ortega Jiménez y P. Cremades García (eds.), Cine y derecho en 13 películas. Alicante: Editorial Club Universitario, 2008, 43-62.

DERrIDA, J., The Ear of the Other. Otobiography, Transference, Translation. Lincoln y Londres: University of Nebraska Press 1985.

Fletcher, G. P., Basic Concepts of Criminal Law. Nueva York: Oxford University Press 1998.

Gentlzer, E., Contemporary Translation Theories. Clevedon: Multilingual Matters 2001

GODARD, B., "Theorizing feminist discourse/Translation», en: Susan Bassnett y André Lefevere (eds.), Translation, History and Culture. Londres: Frances Pinter, 1990, 87-96.

Greenfield, S., G. Osborn y P. Robson, Film and the Law: The Cinema of Justice. Oxford y Portland: Hart 2010.

HAMBLEy, G. E., «The image of the jury in popular culture», Legal Reference Services Quarterly 12-2/3 (1993), 171-216.

Hermans, T., Translation in Systems. Descriptive and Systemic Approaches Explained. Manchester: St. Jerome 1999.

InSDORF, A., Indelible Shadows: Film and the Holocaust. Nueva York: Random House 1983.

KAMIR, O., "Why "Law-and-Film» and what does it actually mean? A perspective", Continuum 19-2 (2005), 255-278.

Latorre Latorre, V., Anatomía de un asesinato. Valencia: Tirant lo Blanch 2005.

Lefevere, A. (ed.), Translation/History/Culture: A Sourcebook. Londres y Nueva York: Routledge 2002.

Levi, R. D., The Celluloid Courtroom: A History of Legal Cinema. Westport: Praeger 2005.

MachurA, S., y P. RoBson, Law and Film. Malden: Blackwell 2001.

MonZÓ NeBot, E., «Derecho y traductología en la formación del traductor jurídico: Una propuesta de formación virtual», Translation Journal 12-2 (2008), 40360-40378.

MuÑoz Conde, F., y M. MuÑoz Aunión, ¿Vencedores o vencidos?: Comentarios jurídicos y cinematográficos a la película de Stanley Kramer 'El Juicio de Nuremberg'(1961). Valencia: Tirant lo Blanch 2003.

Presno Linera, M. A., y B. Rivaya García (coords.), Una introducción cinematográfica al derecho. Valencia: Tirant lo Blanch 2006.

Rivaya García, B., Un vademécum judicial. Cine para jueces. Valencia: Tirant lo Blanch 2012.

Robson, P., «Images of law in the fiction of John Grisham», en: J. Morison y Ch. Bell (eds.), Tall Stories?: Reading Law and Litterature. Aldershot: Dartmouth, 1996, 201-222.

ŠARCEVIC, S., «Translation and the law: An interdisciplinary approach», en: M. SnellHornby, F. Pöhhacker y K. Kaindl (eds.), Translation Studies: An Interdiscipline. Amsterdam: John Benjamins, 1994, 301-308.

Silbey, J. M., «The Politics of Law and Film: Introduction to Symposium on Legal Outsiders in American Film», Suffolk Law Review 42 (2009), 755. 
Soto Nieto, F., y F. J. FernándeZ, Imágenes y justicia: El derecho a través del cine. Madrid: La Ley 2004.

Strickland, R. J., «The Cinematic Lawyer: The Magic Mirror and the Silver Screen», Oklahoma City University Law Review 22-1 (1997), 13-24.

Tyмосzко, M., Translation in a Postcolonial Context: Early Irish Literature in English Translation. Manchester: St. Jerome 1999.

Venuti, L., The Translator's Invisibility: A History of Translation. Londres y Nueva York: Routledge 2008.

Vidal Claramonte, M. C. A., «Re-presenting the "Real”: Pierre Bourdieu and Legal Translation», The Translator 11-2 (2005), 259-275.

\section{Anexo: Listado de películas sobre derecho}

\section{Filosofía del Derecho}

Vencedores o Vencidos de Stanley Kramer (1961) (derecho positivo vs. derecho natural) Los intocables de Brian de Palma (1987) (derecho y justicia)

Sin perdón de Clint Eastwood (1992) (derecho y violencia)

\section{Derecho Constitucional}

Matar a un ruiseñor de Robert Mulligan (1962) (derechos humanos)

Todos los hombres del presidente de Alan J. Pakula (1976) (corrupción política)

Lloviendo piedras de Ken Loach (1993) (derechos sociales)

\section{Derecho Procesal}

Más allá de la duda de Fritz Lang (1956) (pruebas procesales)

Doce hombres sin piedad de Sydney Lumet (1957) (jurado)

Testigo de cargo de Billy Wilder (1957) (testigos)

\section{Derecho Penal}

Soy un fugitivo de Mervin LeRoy (1932) (esencia del derecho penal)

Cadena perpetua de Frank Darabont (1994) (cadena perpetua)

La vida de David Gale de Alan Parker (2003) (pena de muerte)

\section{Derecho Civil}

Veredicto final de Sidney Lumet (1982) (procedimiento civil) El dilema de Michael Mann (1999) (indemnizaciones por daños) 
La heredera de William Wyler (1949) (derecho de sucesiones)

\section{Derecho Mercantil}

Wall Street de Oliver Stone (1987/2010) (mercados bursátiles)

Legítima defensa de Francis Ford Coppola (1997) (cláusulas contractuales abusivas) Perdición de Billy Wilder (1944) (fraude)

\section{Derecho Público Internacional}

Senderos de gloria de Stanley Kubrick (1957) (derecho en tiempos de guerra) La batalla de Argel de Gillo Pontecorvo (1966) (terrorismo y uso de la fuerza) Camino a Guantánamo de Michael Winterbottom y Mat Whitecross (2006) (terrorismo de estado)

\section{Derecho Privado Internacional}

Matrimonio de conveniencia de Peter Weir (1990) (matrimonio de conveniencia) La pequeña Lola de Bertrand Tavernier (2004) (adopciones)

La promesa de Jean-Pierre y Luc Dardenne (1996) (explotación de trabajadores inmigrantes)

\section{Derecho del Trabajo}

La huelga de Sergei M. Eisenstein (1924)

Tiempos modernos de Charles Chaplin (1936)

Ladrón de bicicletas de Vittorio De Sica (1948)

\section{Derecho del Medio Ambiente}

Erin Brockovich de Steven Soderbergh (2000)

La pesadilla de Darwin de Hubert Sauper (2004)

Acción civil de Steven Zaillain (1998) 